\title{
Data Forwarding in Wireless Relay Networks
}

\author{
Tzu-Ming Lin, Wen-Tsuen Chen and Shiao-Li Tsao \\ ITRI, NTHU, ENCTU, \\ Hsinchu, \\ Taiwan, R.O.C.
}

\section{Introduction}

Broadband wireless communication has brought users a number of multimedia services for years. As wireless broadband markets mature, system operators face new problems. First, system capacity is bounded by finite radio resources. Second, service providers increasing spend on network deployments and services provisions. With limited radio resource and increased costs, operators have no choice but to force users to pay higher rates to preserve the same service quality than they had several years ago.

The expectation is that these problems are solved by introducing relay station (RS) into traditional wireless systems. The relaying technology has proven to be a feasible technology to expand system capacity and reduce deployment costs simultaneously. One of the most popular technologies is cooperative communications that boost network throughput significantly by improving the radio quality for transmission and reception (Nosratinia et al., 2004); (Mohr, 2005); (Doppler et al., 2007). Numerical results (Soldani \& Dixit, 2008) showed the cost saving benefits when conventional communication networks adopt relay functionality. From this, a commercial cellular system can save more than $56 \%$ on capital expenditures when RSs are deployed.

RS is a wireless communication station that provides relay services for receiving and forwarding radio signals between two stations. In a cellular system, RS is in charge of receiving, decoding, and forwarding data between the Base Station (BS) and the Subscriber Stations (SSs). During the relaying, RS provides additional features to assist data transmissions. For example, (Tao et al., 2007) proposed a novel data forwarding scheme in this environment. The novel data forwarding scheme improved the transmission efficiency by approximately $66 \%$.

Although data relay shows superior performances in throughput enhancements and cost savings. Multi-hop relaying introduces new issues that impact overall performance. To indicate a routing path in multi-hop circumstances, additional control information should be introduced. The routing indication design for multiple radio links introduces new overhead and may impact transmission efficiency during relay. Imprecise indications may waste RS computation and storage since it does not know what it shall relay and where to forward. Moreover, there may be a case that data destined to others are received by the RS that is not in charge. Imprecise relay indication will not only increase overheads but also lead RS to use its processor and storage inefficiently. In other words, data forwarding with ambiguous control information would increase RS complexity and buffer storage unnecessarily. During 
the relaying, RS shall forward data as simple as possible to prevent wasting processing power and storage. This study proposes a burst-switch concept aiming to tackle the issues and provides a simple and efficient data forwarding for wireless relay networks.

The rest of the chapter is organized as follows. First, wireless relaying and a conventional relay system are overviewed in section 2 . The proposed new forwarding mechanism is then elaborated in section 3; section 4 presents the evaluation and simulation results for the mentioned issues. At last, conclusions are given in section 5 .

\section{Background and related works}

Relay technology has been investigated for years, and a realistic relay system will be deployed in few years to enhance legacy wireless networks. This section overviews IEEE 802.16 communication system and introduces the relay enhancements. The data forwarding mechanisms adopted in the system are also discussed to address the issues.

\subsection{Overview of wireless relay networks}

A wireless relay network consists of a BS, one or more RSs, and numbers of SSs. In the network, directly or through the assistances of RSs. BS forwards the downstream data coming from outside network to SS while RSs relay upstream data generated by SSs to BS. Since all the data transmissions within the network are arranged by BS and there are no communications between RSs, the relay network is usually constructed as a tree topology, which is illustrated in figure 1.

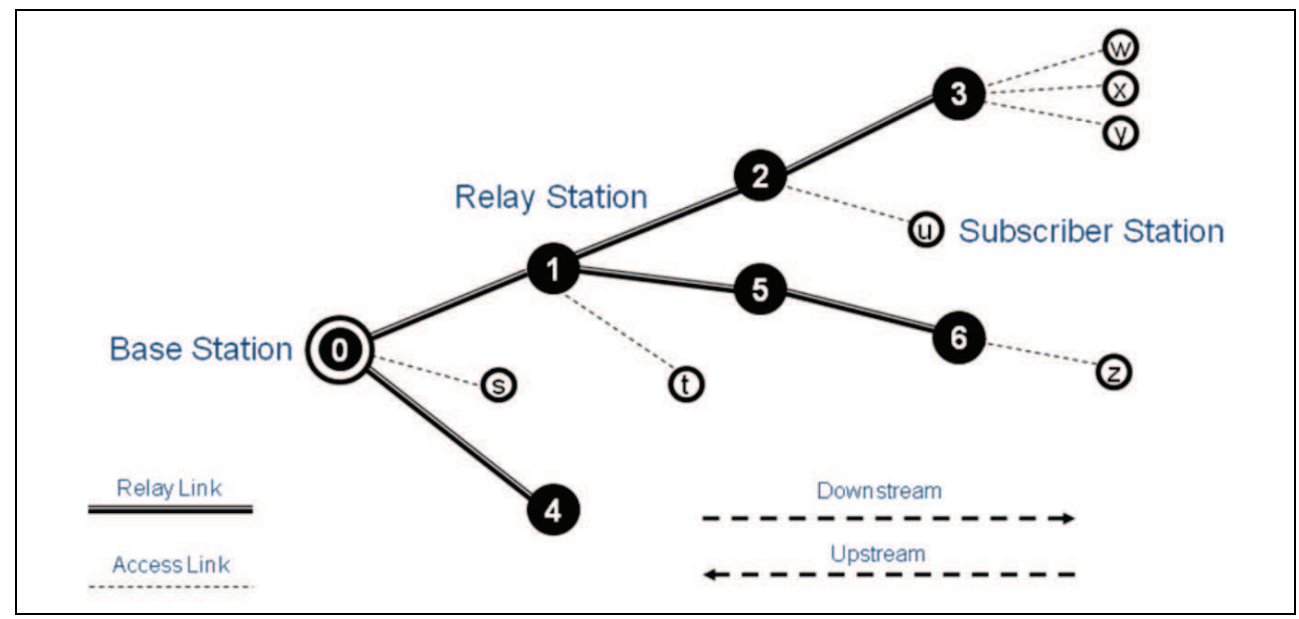

Fig. 1. Wireless Relay Network

There are two types of radio links in this network: relay link and access link. The radio link between a BS and a RS and between two RSs are called relay links, and BS constructs a relay path by multiple relay links. The access link is the radio communication between a SS and its access station, which can be a BS or an access RS. The access RS is a RS attached by a SS and can helps BS for relaying data to the SS. For the example in figure $1, \mathrm{RS}_{2}$ is an access $\mathrm{RS}$ of $\mathrm{SS}_{\mathrm{u}}$ and assists $\mathrm{BS}_{0}$ to provide relay services for $\mathrm{SS}_{\mathrm{u}}$. $\mathrm{BS}_{0}$ allocates resources 
along the relay path between $\mathrm{SS}_{\mathrm{u}}$ and itself so that the help on data relay in corresponding relay links.

\subsection{IEEE 802.16 and multi-hop relay network}

IEEE Std $802.16 \mathrm{e}^{\mathrm{TM}}-2005$ is one of the most popular wireless broadband networks nowadays, and figure 2 shows the reference model for that system. The system consists of two layers, Medium Access Control (MAC) and Physical (PHY) layers, to handle wireless communications. Packets from TCP/IP layer are translated into MAC Protocol Data Units (MPDUs) and then encoded into a PHY burst. The burst is associated with a MAP Information Element (MAP-IE) that indicates a station for receiving and decoding the burst. After the data process, BS transforms both the burst and the associated MAP-IE into a radio frame and pumps it into wireless medium.

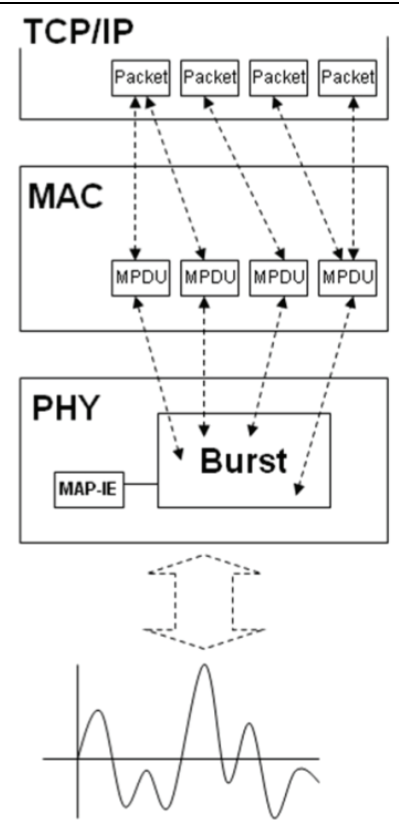

Fig. 2. Data Processing in IEEE 802.16

The overview of 802.16e frame structure is depicted in figure 3. The frame composes two subframes: downlink and uplink subframes, and starts with a synchronization part of preamble and Frame Control Header $(\mathrm{FCH})$. The first part is used for each receiving station synchronize with BS and abstracting the frame. Following the synchronization part, the frame header further includes a downlink MAP (DL-MAP) and an uplink MAP (UL-MAP), which consists of MAP-IEs to indicate the stations where and how to access data bursts. As stated before, each data burst is associated with an MAP-IE, and one or more MPDUs destining to a destination can be concatenated or packed into the burst. With a connection identity (CID) in the MAP-IE, every receiving station locates and receives MPDUs in the desired PHY burst, and has no need to check all the bursts in the frame. 


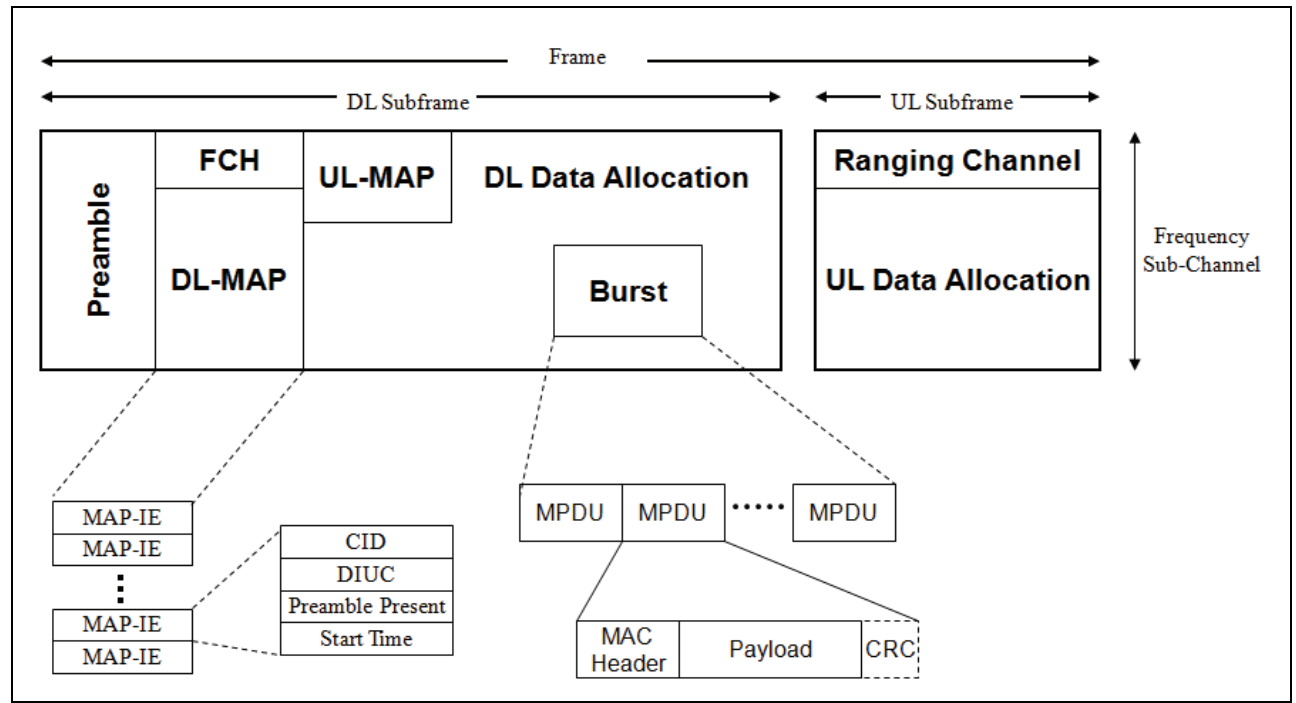

Fig. 3. IEEE 802.16e Frame Structure

The system further specifies an option for disabling MAP to save overheads so that more data can be allocated. In this case, receiving stations should put more efforts to process entire frame since there are no indications in frame header any more. Without MAP indication, the receiving station cannot but store the whole frame to check if there are any desired MPDUs. However, it is inefficient for buffering and checking all the MPDUs in a frame. Although, the operations brought overheads but the problem should not be as serious as that in multi-hop communications. Because of redundant processing and transmissions during relay, multi-hop data forwarding makes the overhead become a severe problem.

\subsection{Data forwarding and issues in 802.16j MR network}

IEEE working group specifies Multi-hop Relay (MR) support for 802.16e system in IEEE Std 802.16j ${ }^{\mathrm{TM}}$-2009. The specification aims to solve the capacity problem and reduce development cost with advanced relay technologies. Efficiency during data relay is also a major concern for implementing RSs. Two data forwarding schemes are specified to facilitate relay functionalities and reduce overheads.

The first forwarding scheme is CID-based transmission, in which RS forwards MPDUs based on the CIDs contained in the MAP-IE or MPDU headers. For saving signalling overheads, relayed MPDUs do not carrier any extra routing information, and are transmitted as in 802.16e conventional system. When MPDUs are relayed, each receiving RS gets CIDs from MAP-IEs and checks associating bursts if there are any data required for further forwarding. The RS discards the burst that is not indicated by the recorded CIDs in its forwarding list. When the CID of the burst is in the forwarding list, the receiving RS forwards the burst to the station in next hop. Besides, there is another implementation that RSs forward MPDUs by identifying CIDs containing in MPDU headers. As mentioned, each RS has to process all the MPDU in receiving frame, and determines the MPDUs for relay. Figure 4 depicts the example for this forwarding scheme based on the relay network in 
figure 1. $R S_{1}$ receives all the packets containing in first frame and stores the MPDUs destining to $\mathrm{RS}_{1}, \mathrm{RS}_{2}, \mathrm{RS}_{3}, \mathrm{RS}_{5}$, and $\mathrm{RS}_{6}$. In second frame, $\mathrm{RS}_{2}$ caches the MPDUs from $\mathrm{RS}_{1}$ and checks which MPDUs it shall relay. The data for $\mathrm{RS}_{2}$ and $\mathrm{RS}_{3}$ are received and only $\mathrm{RS}_{3}$ data are relayed. After that, $\mathrm{RS}_{3}$ receives the data by checking MPDU headers and performs relaying for $\mathrm{SS}_{\mathrm{w}}, \mathrm{SS}_{\mathrm{y}}$, and $\mathrm{SS}_{\mathrm{y}}$ individually. Moreover, the first option for adopting MAP-IE indications can filter unnecessary bursts before looking into every MPDU and RS needs not store all the data in the frame. Otherwise, checking CIDs in MPDU headers would force RSs to receive all the data in a frame. As a result, the using of MAP is a trade-off issue between storage and signalling because enabling MAP prevents RSs to store MPDUs unnecessarily but brings extra control overheads.

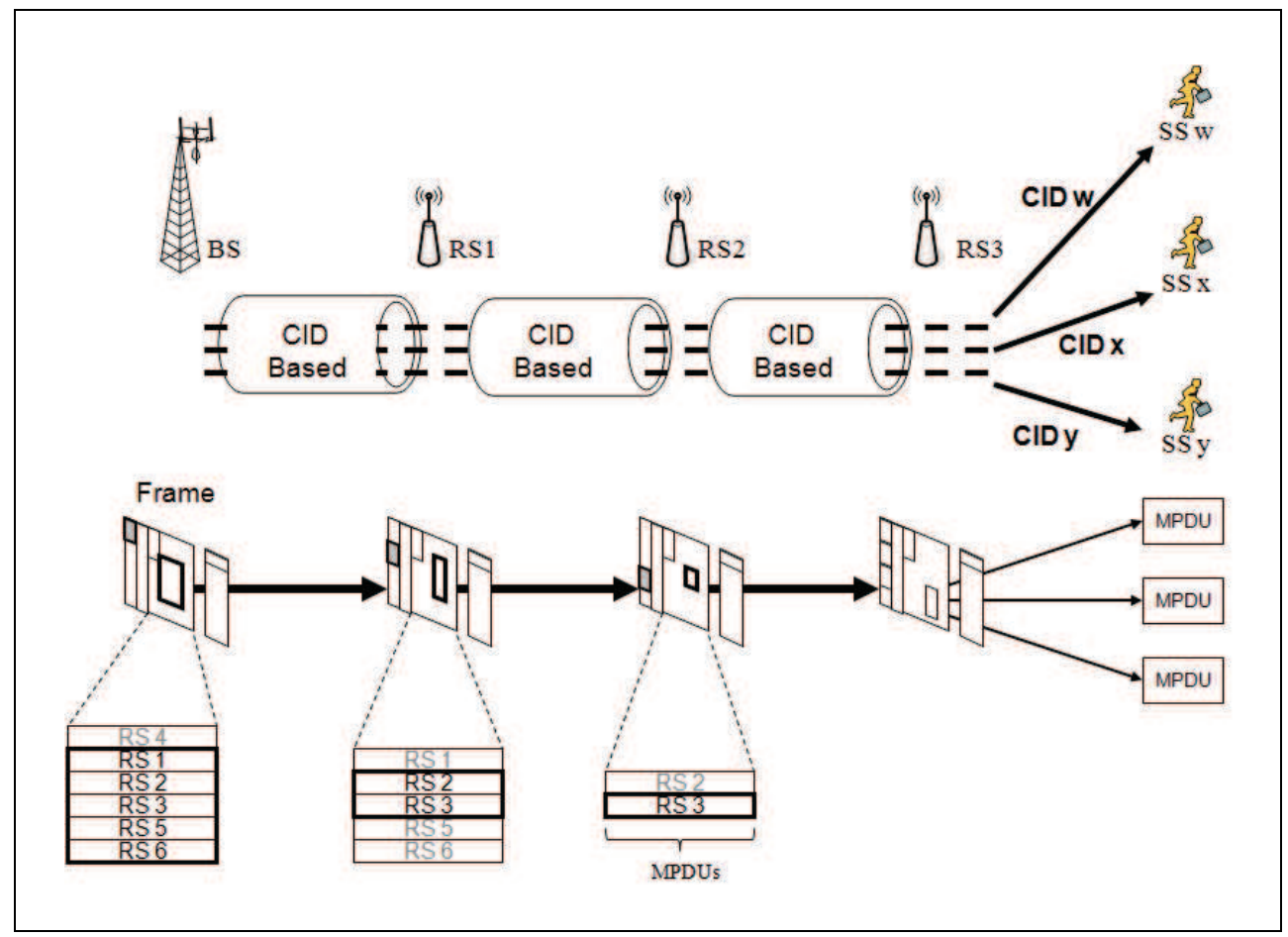

Fig. 4. CID-Based Transmission

The second scheme is tunnel-based transmission, in which BS and access RS encapsulates MPDUs into a Tunnel PDU (T-PDU) and transmits these data through a tunnel in between. Figure 5 shows an example of this scheme. BS, the ingress station of a tunnel, aggregates MPDUs into one or more T-PDUs, and transmits the data to an access RS. The access RS at the egress of tunnel, e.g. $\mathrm{RS}_{3}$, is responsible for removing tunnel headers and forwarding the decapsulated MPDUs to SSs. Besides, the intermediate RSs along a relay path relay the TPDU to the tunnel end through the indication of tunnel headers. As CID-based operation, enabling MAP can help RSs to filter unwanted data, and disabling MAP would force RSs to buffer and process all the T-PDUs in the frame. Tunnel header benefits in preventing redundant processes for the group of MPDUs destining to same access RS. However, the 
extra overhead brought from the tunnel header should be considered. Furthermore, the impact caused by adopting MAP and tunnel headers at the same time shall be also investigated.

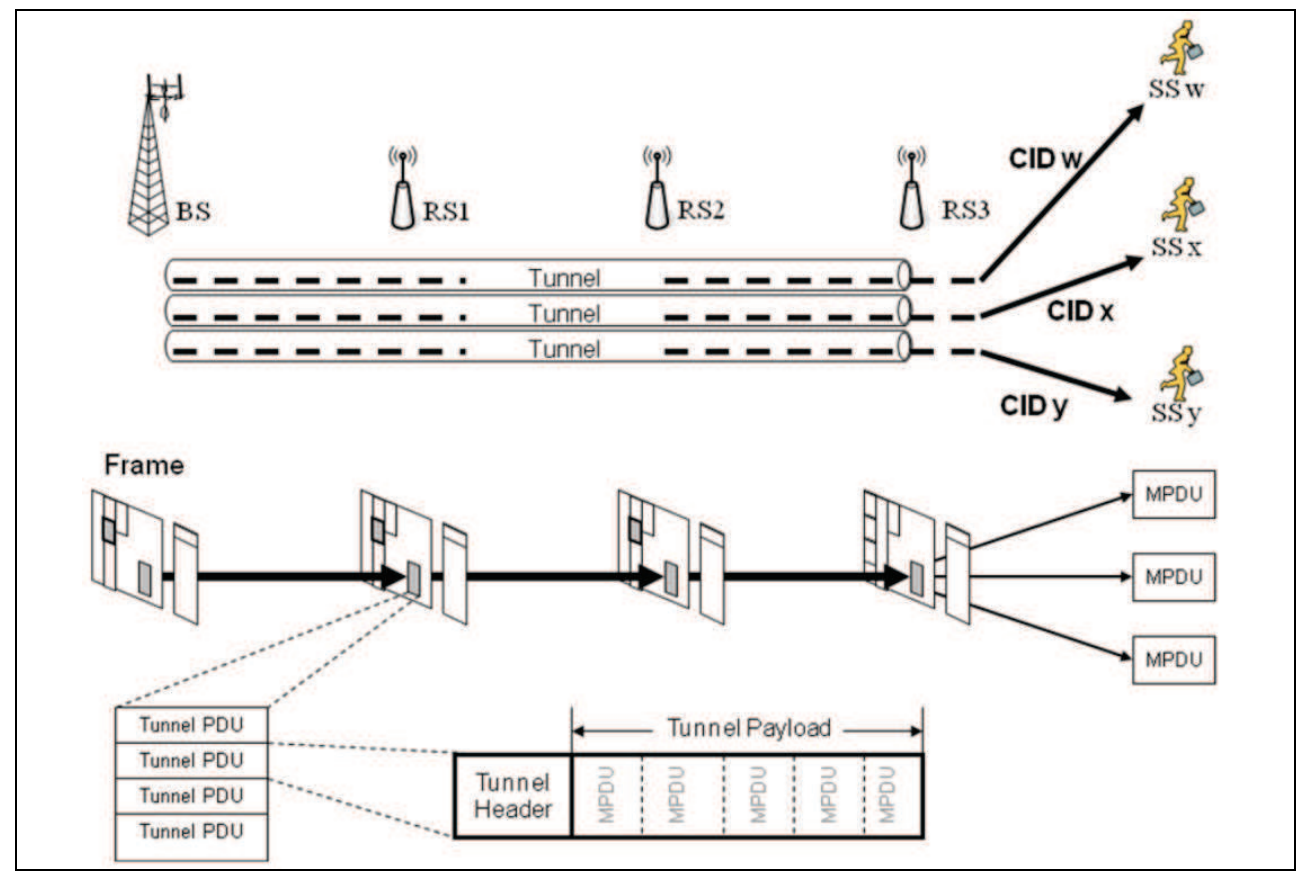

Fig. 5. Tunnel-based Transmission

Comparing these two forwarding schemes, RSs applying tunnels forward data efficiently since RS identifies a MPDU group by a tunnel header in replace of multiple MPDU headers. CID-based scheme provides the forwarding as simple as that in legacy one-hop system, and introduce no extra headers. Although both the two mechanisms can assist data forwarding in multi-hop environments, the overheads caused by excess header processing and unnecessary data buffering shall be discussed in advanced. First, the processing overhead for RSs would rise with the system traffic load. Take the example in figure $1, \mathrm{RS}_{5}$ shall process $2 n$ tunnel headers in tunnel-based scheme when each RS maintains $n$ tunnels. If $\boldsymbol{m}$ MPDUs are scheduled for each SS in CID-based scheme, $4 m$ MPDU headers will be handled by $\mathrm{RS}_{2}$. In the example, both $\boldsymbol{m}$ and $\boldsymbol{n}$ increase with the traffic load and all the intermediate RSs suffer. The impacts to processing complexity of the two schemes are:

$$
\begin{gathered}
C_{\text {Tunnel_based }}=L_{\text {tunnel_header }} \times N_{\text {tunnel }} \times N_{R S} \\
C_{\text {Station_based }}=L_{M P D U_{-} \text {header }} \times N_{M P D U}
\end{gathered}
$$

where $C_{\text {Tunnel_based }}$ and $C_{C I D \_b a s e d}$ define the computation in bits, $L_{\text {tunnel_header }}$ and $L_{M P D U_{-} \text {header }}$ denote the lengths of tunnel and normal MPDU headers, $\boldsymbol{N}_{\text {tunnel }}$ is the tunnel number that a RS shall handle, $N_{R S}$ is the summation of all the RSs behind a receiving station for a relay 
path, and $N_{M P D U}$ is the amount of MPDUs that needs to be forwarded. In a stationary relay network, both $C_{\text {Tunnel_based }}$ and $C_{C I D \_b a s e d}$ grow with $N_{\text {tunnel }}$ and $N_{M P D U}$, and both the two numbers correspond to the network load. As system load rises, tunnel numbers would increase with provisioned connections. If CID-based forwarding is applied in this case, the amount of processed MPDUs would also grow with the increased connections.

Although tunnel header prevent RSs to process MPDU header redundantly, the issue of inefficient data processing and buffering remains unsolved; every RS needs to store all the T-PDUs or MPDUs in a frame before getting relay information. Some undesired data would still be processed and buffered before being processed. If MAP is applied, RSs could handle fewer data since the bursts for other destinations can be filtered by associating MAP-IEs. However, another issue for excess processing is unsolved because there are two-levels of control information, one for MAP and the other for MPDU or T-PDU headers. No matter which mechanism is applied, the addressed problems cannot be taken over totally. Aiming this, a simple data forwarding mechanism is proposed in this study for relaying data more efficiently.

\section{End-to-end burst switch and proposed network model}

This study brings out a new concept of switching burst to forward data in the wireless relay network. The burst switch mechanism uses RSs more efficiently by reducing processed control information and buffered data. Besides, a new network model for adopting the concept is also proposed to realize the forwarding process.

\subsection{End-to-end burst switch}

This literature suggests using an end-to-end burst CID in the associated MAP-IE to forward relayed data, and proposes to relay data with a unique identifier for each relay path in burst level. To reduce carried routing information, data goal to same destination RS are assigned with one burst CID. With the help of the burst CID, intermediate RSs relay bursts without checking the MPDU so as to eliminate the processing in MPDU level. Moreover, checking CIDs in MAP also saves unnecessary processing for the data destining to other destinations. Before relaying data, BS identifies the access RS and sets up the forwarding path for a SS. As the legacy schemes, a data burst binding a relay path aggregates multiple MPDUs and is transmitted in frames. Since each relay path is identified by a burst CID, each RS along a relay path checks the MAP to locate the relayed burst. For the RS not in the path, it just ignores the data burst after checking the MAP. During the relay process, RSs need not to look into MPDUs to identify the data or routing. The usage of CIDs in this scheme is similar to that used in tunnel-based scheme, but the CID is used in burst level, not in T-PDU level. Such an enhancement saves the overheads for both control and data parts. By referring burst CID in frame header, the RS can decide to receive the associated burst or not. If the burst for the relay path is located, the RS transmits the entire burst toward next RS without processing the data in further. The intermediate RSs do not decapsulate inside data but switch the burst along the relay path until the destination RS receives it. After the burst is switched to the end of relay path, the access RS forwards the data to SSs using individual CIDs in access links as BS does in the conventional one-hop network. Since bursts are identified and switched by the burst CID, the proposed forwarding scheme is so called burst switch. 


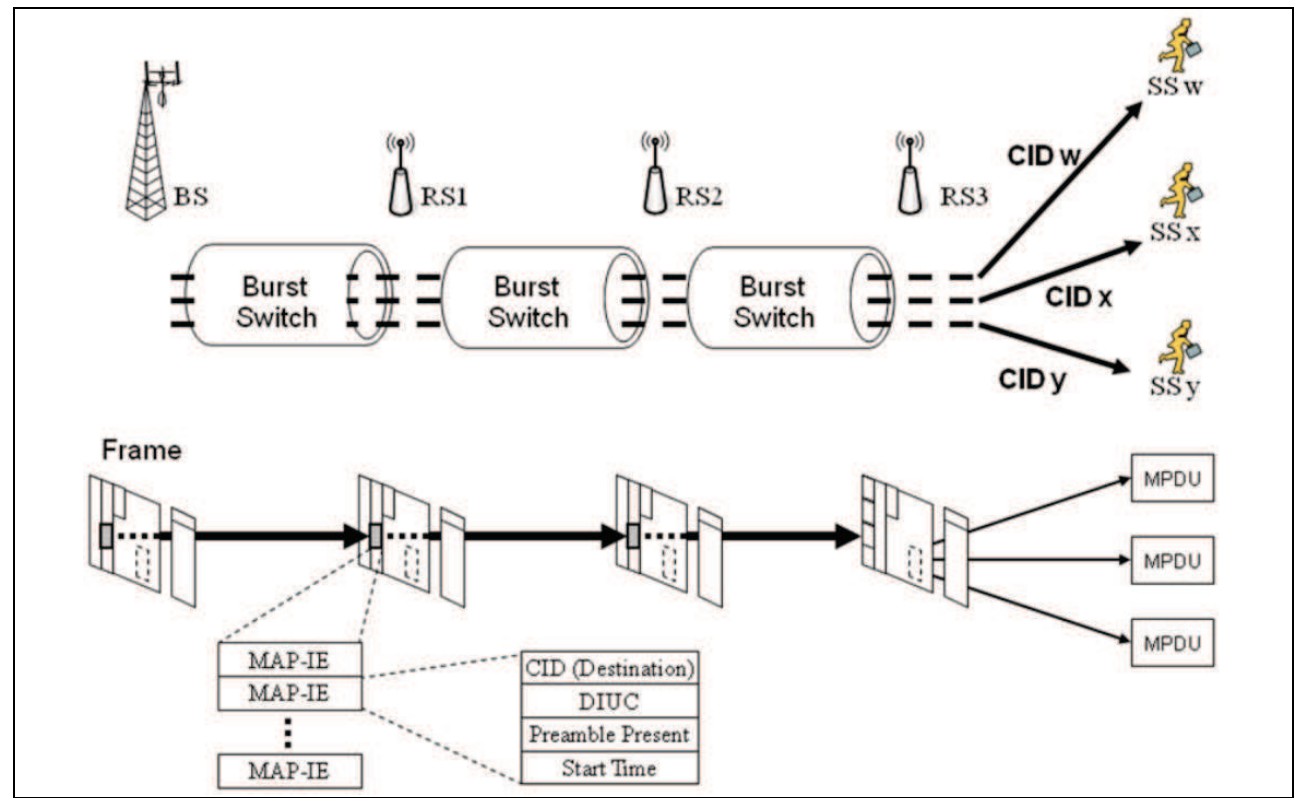

Fig. 6. Burst Switch Transmission

Figure 6 shows the proposed scheme in detail. In the figure, BS establishes a path for switching the burst for $\mathrm{SS}_{\mathrm{w}}, \mathrm{SS}_{\mathrm{x}}$, and $\mathrm{SS}_{\mathrm{y}}$, and assigns an end-to-end burst CID for the relay destination, $\mathrm{RS}_{3}$. When receiving the MAP in first hop, $\mathrm{RS}_{1}$ and $\mathrm{RS}_{2}$ check the burst CID and buffer the burst for switching in second hop. After receiving the switched burst, $\mathrm{RS}_{3}$ decapsulates the burst and processes the MPDUs to see where the destination for the data is. After that, access $\mathrm{RS}_{3}$ forwards the decoded MPDUs to $\mathrm{SS}_{\mathrm{w}}, \mathrm{SS}_{\mathrm{x}}$, and $\mathrm{SS}_{\mathrm{y}}$ separately. The computation for this relay process is:

$$
C_{\text {Burst_switch }}=L_{\text {MAP-IE }} \times N_{R S}
$$

Where $C_{\text {Burst_Switch }}$ is the computation cost, $L_{M A P-I E}$ denotes MAP-IE length and $N_{R S}$ represents the numbers of RSs behind the station. Since $N_{R S}$ is a fixed value as network has been setup, neither the network load nor other traffic factors impact $C_{\text {Burst_switch. }}$. Compared with the tunnel-based and CID-based solutions, the proposed scheme adopts the 32 bits MAP-IE and saves $33 \%$ overhead while the two schemes apply 48 bits T-PDU or MPDU headers. Therefore, it can be expected that $C_{\text {Burst_Switch }}<C_{\text {Tunnel_based }}<C_{\text {CID_based }}$. Moreover, RS with burst switch identifies relay destination before storing data and takes the advantage of storage saving.

\subsection{Proposed network model}

Before providing relay services, the relay system shall construct a relay network model to determine relay paths for all the SSs. This paragraph provides an overview of the proposed model, in which the burst switch mechanism can be applied. In the network, BS maintains a forwarding table that keeps the CID for every relay path and access link while RSs only record the CIDs for the stations in behind. The CIDs includes the burst CID of access RSs 
and also the access CIDs of SSs. Figure 6 shows the model for the example of the wireless relay network in figure 1.

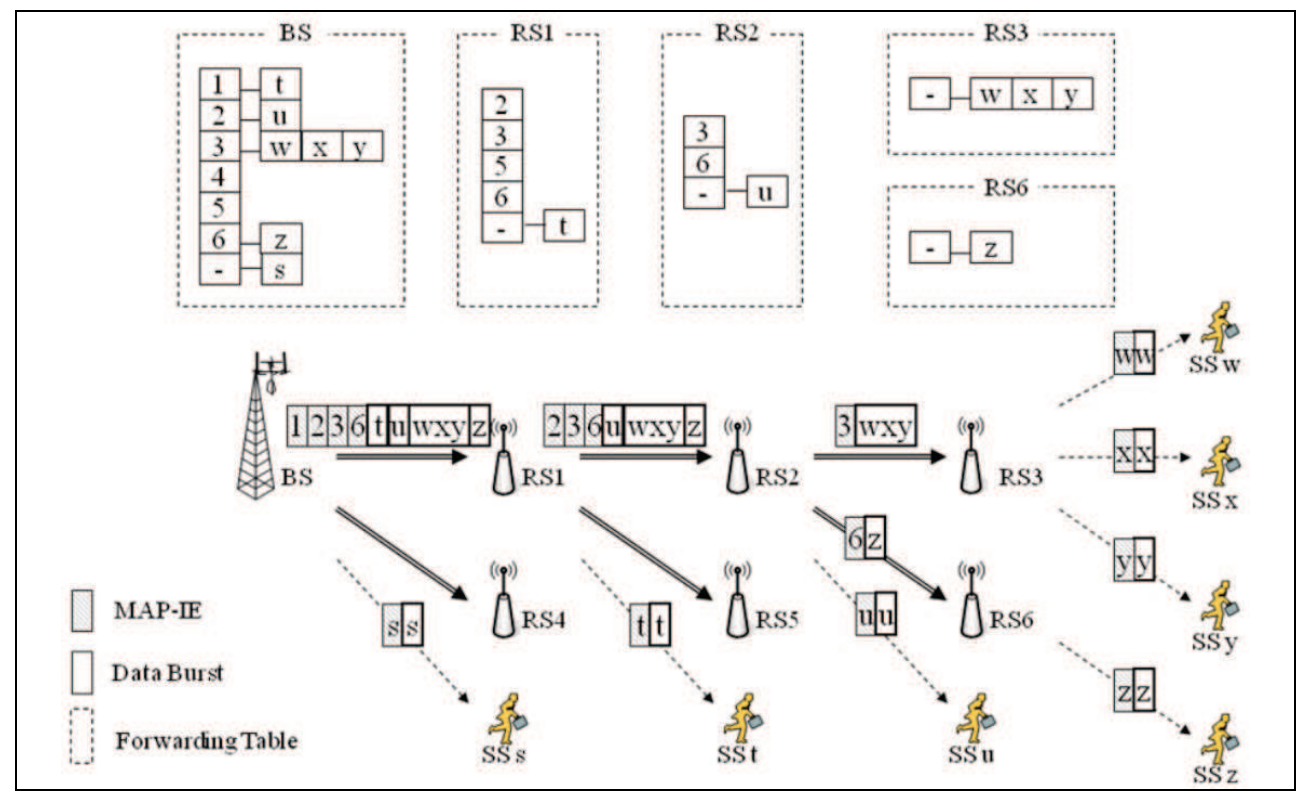

Fig. 7. Proposed Relay Network

In the example, BS can obtain the CIDs for $\mathrm{RS}_{1}$ to $\mathrm{RS}_{6}$ and $\mathrm{SS}_{\mathrm{t}}$ to $\mathrm{SS}_{\mathrm{z}}$ from the table, and $\mathrm{RS}_{2}$ only holds the CIDs for $\mathrm{RS}_{3} \mathrm{RS}_{6}$, and $\mathrm{SS}_{\mathrm{u}}$. When initializing a relay transmission, BS first identifies a receiving SS and the associating access RS from the table. Then, radio resources are allocated for relay links in the relay path. By the MAP indications, intermediate RSs switch burst by checking the CIDs within MAP when receiving a frame. Only when the received burst CID matches the recorded CID, the binding burst is switched to next hop. Intermediate RSs switch the burst until the burst arrivals the relay destination, and the access RS forwards MPDUs to SSs in access links after receiving.

In figure 6, data bursts for all the SSs are ready for relay, and BS assigns burst CID for each burst based on the forwarding table. Bursts for $\mathrm{SS}_{\mathrm{t}}$ associates with the CID of $\mathrm{RS}_{1}$, and bursts for $\mathrm{SS}_{\mathrm{u}}$ and $\mathrm{SS}_{\mathrm{z}}$ are associated with the CIDs of $\mathrm{RS}_{2}$ and $\mathrm{RS}_{6}$ respectively. Because $\mathrm{SS}_{\mathrm{w}}, \mathrm{SS}_{\mathrm{x}}$, and $\mathrm{SS}_{\mathrm{y}}$ attach the same access RS, data for these SSs are encapsulated together and assigned with the CID of $\mathrm{RS}_{3}$. When the relaying begins, $\mathrm{RS}_{1}$ checks CIDs in the MAP and see if there are any bursts associating with the CIDs of $\mathrm{SS}_{\mathrm{u}}, \mathrm{RS}_{2}, \mathrm{RS}_{3}$, and $\mathrm{RS}_{6}$. Likewise, $\mathrm{RS}_{2}$ operates similarly, but the difference is switching bursts to $\mathrm{RS}_{3}$ and $\mathrm{RS}_{6}$ individually due to separated burst CIDs. After receiving the bursts, $\mathrm{RS}_{3}$ and $\mathrm{RS}_{6}$ forward the data to their SSs using the $\mathrm{CIDs}_{\mathrm{w}} \mathrm{SS}_{\mathrm{w}}, \mathrm{SS}_{\mathrm{x}}, \mathrm{SS}_{\mathrm{z}}$, and $\mathrm{SS}_{\mathrm{u}}$ to complete the relay process.

The major outcomes of the proposed scheme are determined control overhead, simple forwarding, and efficient buffer usage. Unlike tunnel-based and CID-based approaches, processing overheads of proposed method increase with static RS number, not dynamic network traffic load. Moreover, it is simple because each RS identifies desired data by matching CIDs in frame header. Beside, RSs identify relay destination before caching it, and 
do not store data irrelevantly. To validate the outcomes, next section evaluates the performance of burst switch among various scenarios and compares the results with the other forwarding schemes.

\section{Simulation results}

This study investigates the issues on data forwarding during relay in terms of process and storage efficiency. Two metrics are introduced for the evaluations. Processing overhead, the first metric, is the total bits that RS shall handle through all the relay process. The second metric, storage overhead, is the memory space in which RS needs to store bursts during relaying. Besides, five forwarding schemes are evaluated in the simulations: (1) CID_w_MAP, (2) CID_w/o_MAP, (3) Tunnel_w_MAP, (4) Tunnel_w/o_MAP, and (5) Burst Switch. Schemes (1) and (3) represent CID-based and tunnel-based forwarding schemes with MAP support while scheme (2) and (4) denote the relay schemes without MAP indications. For the schemes enabling MAPs, the CID in a MAP-IE directs the station in a hop-by-hop manner, which means RSs swap CIDs for each hop during the relaying. The proposed burst switch scheme employs a burst CID to indicate an end-to-end relay path for each access RS. Table 1 lists detailed parameters, including system and traffic, and the four traffic types defined in 802.16e are also used as inputs to show the performance differences between services.

\begin{tabular}{|l|c|l|c|}
\hline \multicolumn{2}{|c|}{ System Parameters } & \multicolumn{2}{c|}{ Traffic Parameters } \\
\hline Channel Bandwidth & $20 \mathrm{MHz}$ & Arrival Time - UGS & $30 \mathrm{~ms}$ \\
\hline Frame Duration & $5 \mathrm{~ms}$ & MPDU Size - UGS & 200 bytes \\
\hline Symbols Per Frame & 48 & Arrival Time - RTPS & $30 \mathrm{~ms}$ \\
\hline FFT Size & 2048 & MPDU Size - RTPS & 1000 bytes \\
\hline Code Rate & $1 / 2$ & Arrival Time - NRTPS & $100 \mathrm{~ms}$ \\
\hline Modulation & QPSK & MPDU Size - NRTPS & 1500 bytes \\
\hline RS Number & 20 & Arrival Time - BE & $300 \mathrm{~ms}$ \\
\hline SS Number & 100 & MPDU Size - BE & 800 bytes \\
\hline
\end{tabular}

Table 1. Simulation Parameters

Figure 8 illustrates the statistics of processing overhead for RSs in 2-hop relay case. It is observed that CID_w/o_MAP makes severe processing overheads. Moreover, the curve grows dramatically as the number of traffic flows increases. On the contrary, the overheads for the other forwarding schemes rise slightly. The figure tells that disabling MAPs in twohop case would cause excess overhead for RSs and the results come from processing all the MPDUs in a frame. Although MAP is also disabled in scheme (4), Tunnel_w/o_MAP performs best by introducing tunnel header to avoid the drawback. When comparing with scheme (4), Tunnel_w_MAP shows a little worse performance since MAPs and tunnel headers are provided redundantly. It is worth to note that $C I D \_w \_M A P$ and proposed scheme show identical results in the figure because the CIDs in MAPs both denote the destination RS in two-hop cases. 


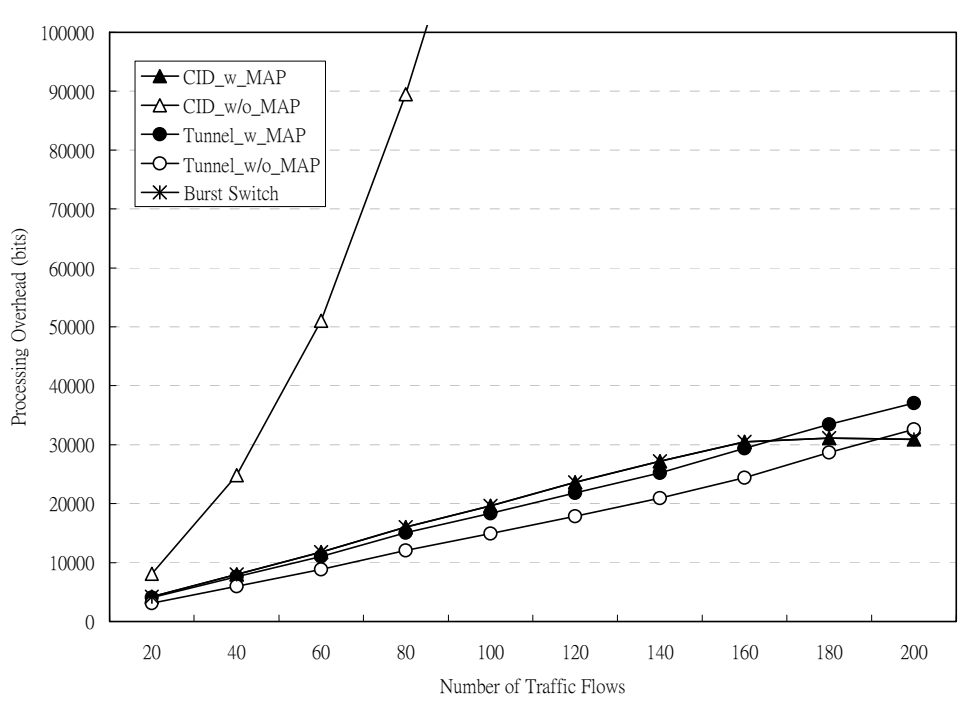

Fig. 8. Processing Overhead in 2-hop Scenario

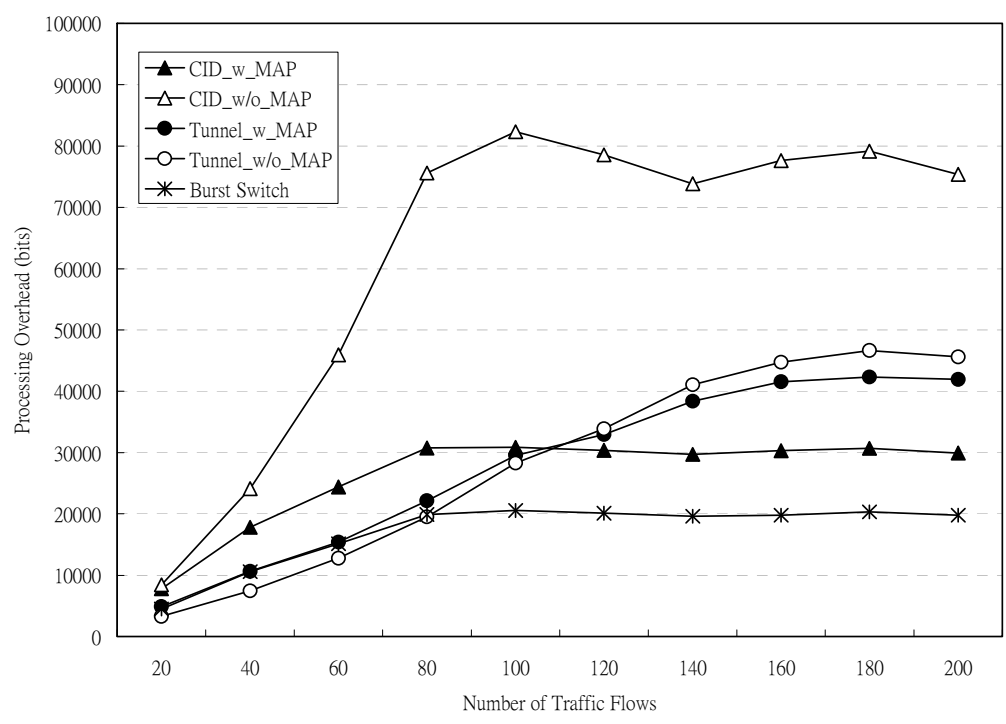

Fig. 9. Processing Overhead in 5-hop Scenario

As the relay hop count increases, RSs shall handle more and more control information in the relaying process, and figure 9 depicts the results in multi-hop relay environments. Generally speaking, the processing overheads of all the schemes increase with traffic load in light traffic load cases (i.e. less than 80 traffic flows). Tunnel-based schemes have less 
computation overhead than CID-based schemes since tunnel headers can prevent RSs from processing all the MPDUs in a frame. Nevertheless, the curse of tunnel-based schemes also rises as increased traffic in heavy traffic load conditions (i.e. more than 100 traffic flows), and it is because more and more tunnels are established as traffic load increases. Besides, high traffic load also increases the MPDU amount, and, unfortunately, CID_w/o_MAP still obtains the worst results. The key worth to be noticed here is that the curve of $C I D \_w \_M A P$ and Birth Switch scheme rises limitedly in heavy load cases. Compared with the CID_w_MAP, RSs employing Birth Switch save about $33 \%$ overhead in processing MPDUs, and the figure also shows the outcome. As a consequence, it is obtained that schemes (2), (3), and (4) are impact by system load, and the processing overhead increases with the number of traffic flows. CID_w_MAP and the proposed scheme can provide a stable outcome in RS processing, especially when the relay network is loading high. The burst switch concept can save more overheads than $C I D \_w \_M A P$, and is suitable to forward data in multi-hop environments.

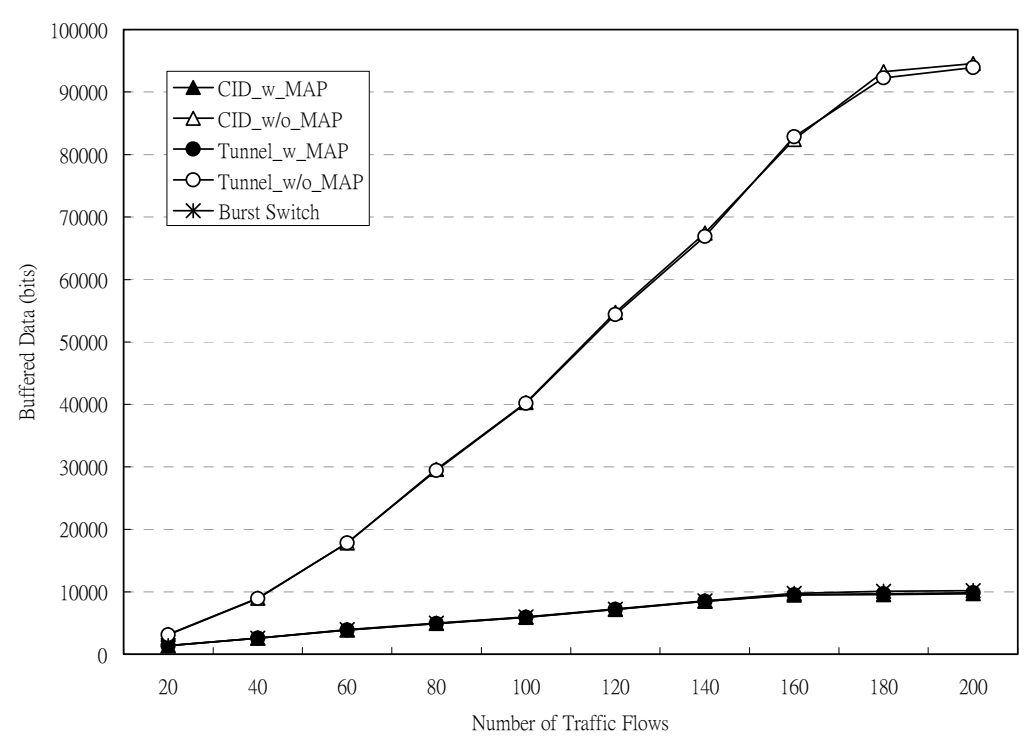

Fig. 10. Storage Overhead in 2-hop Scenario

The second issue is storage overhead, and this study use figure 10 and figure 11 to discuss the evaluations. Both the two simulations confirm the impact resulting from traffic loads. In light load conditions, RSs in 2-hop case do not buffer as much data as those in 5-hop case because the amount of relayed data is small. When the traffic loads high, RSs in both cases have to forward and store more data. Note that the curve becomes saturated for high load cases in figure 10 due to limited radio resource. Moreover, it is easy to find that the schemes without MAP indication, e.g. (2) and (4), cache much more data than the other three schemes do. It is because RSs cannot identify MPDUs before receiving the entire frame, and hence check the MPDUs one by one. On the contrary, RSs in other schemes can filter MPDUs by either frame or tunnel headers so that data would not be buffered inadequately. 


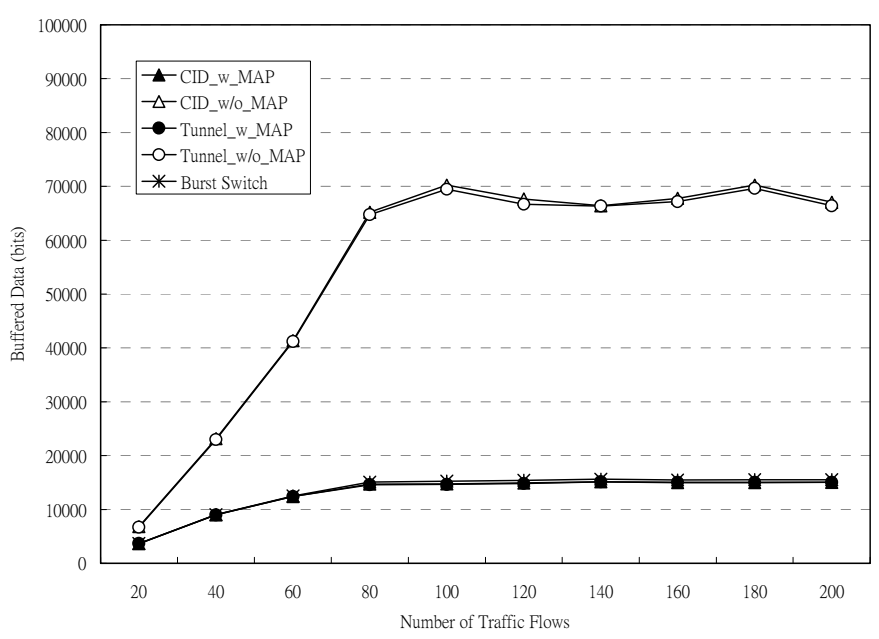

Fig. 11. Storage Overhead in 5-hop Scenario

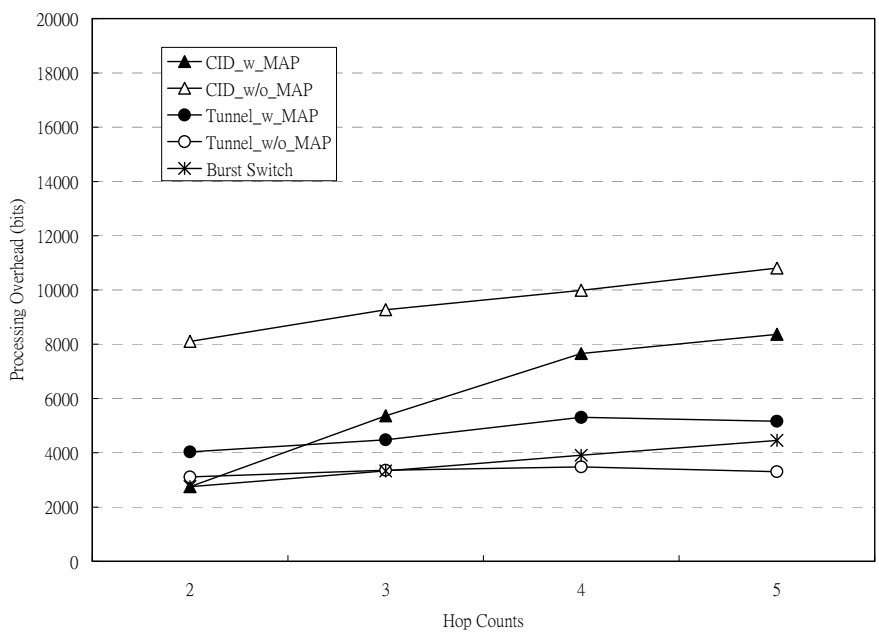

Fig. 12. Processing Overhead in Light Load Cases

This study further evaluates the impact brought from long path lengths. Figure 12 and 13 show the results in light and heavy traffic load conditions respectively. In the simulations of figure 12, the scheme, CID_w/o_MAP, performs worst in all the cases. With the MAP assistance, $C I D \_w \_M A P$ can perform better than tunnel-based solutions. However, the tunnel solution without MAP outperforms the other tunnel-based scheme because of avoiding extra processing for both MAP and tunnel headers. Furthermore, the RS 
employing burst switch also avoids redundant and unnecessary processing by burst CIDs so that the proposed scheme also has superior performance in light load conditions.

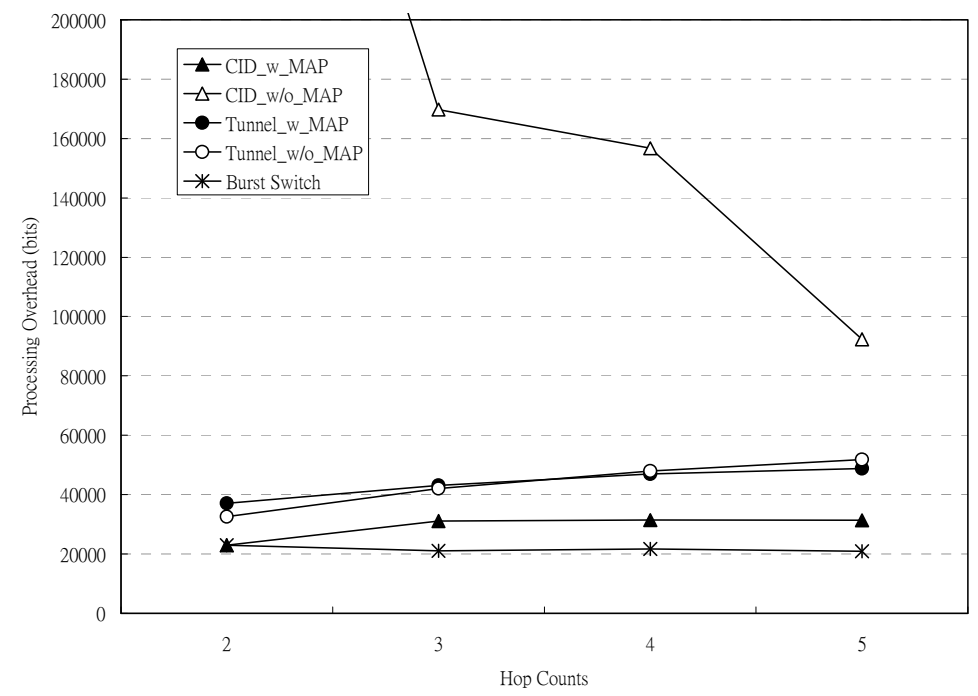

Fig. 13. Processing Overhead in Heavy Load Cases

In figure 13, these forwarding schemes show similar result with that in previous experiment. CID-based forwarding schemes show the frustrated results in heavy load occasions if the network disables MAP support. The other four mechanisms obtain more stable performance in overhead saving when relay hop count increases. Regarding tunnel-based solutions, it is found that enabling MAP does not increase as much overheads as applying tunnel headers because it is tunnel header to dominate the overhead in heavy load conditions. The forwarding schemes without introducing any extra MPDU headers perform better in the simulation. The simulations also suggest the best performance of Burst Switching in multihop environments, especially for heavy load cases.

Processing complexity and storage usage are critical issues for RS developments in wireless relay networks. The evaluation results depict overall comparison of the two metrics in figure 14 and figure 15. We can see the performance of overhead saving among the data forwarding schemes. This study uses the results of Tunnel_w/o_MAP as basics to show following observations. (1) Without MAP indications, CID-based forwarding scheme performs worst in saving processing and storage overheads, no matter in 2-hop or 5-hop cases. (2) In tunnel-based schemes, enabling MAP indication can reduce the amount of cached data with some penalty from extra MAP-IE processing. (3) Comparing the benefits coming from applying MAP and tunnel headers, CID_w_MAP outperforms Tunnel_w/o_MAP in storage efficiency since unnecessary MPDU buffering is avoided. (4) CID_w_MAP scheme introduce irrelevant processing overhead in 5-hop and light load case although it shows more adequate results than the other three conventional schemes. (5) The proposed burst switch scheme performs better than all other forwarding schemes in both 
heavy and light traffic load conditions. (6) Considering the performance among different traffic loads, burst switch scheme saves approximately $8 \%$ computation and about 60 73\% storage costs from the results of evaluations.

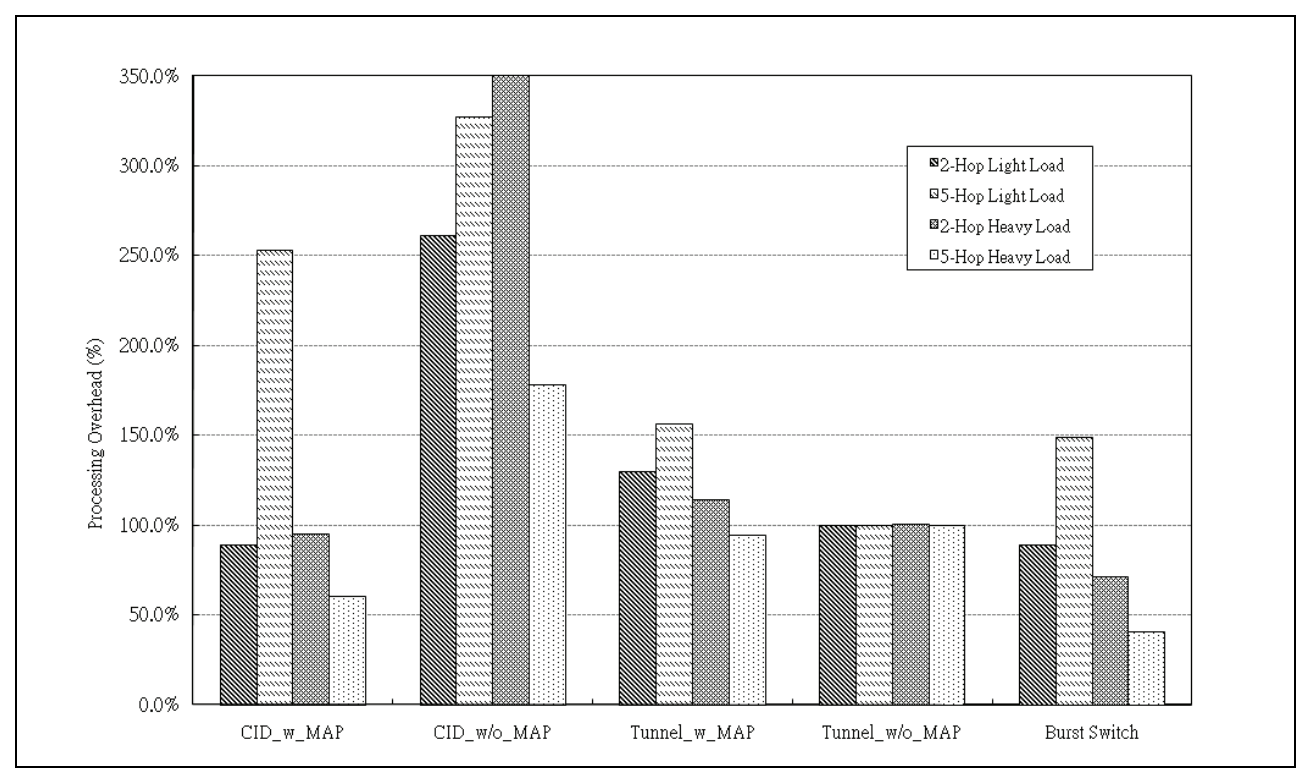

Fig. 14. Overall Comparison for Processing Overhead

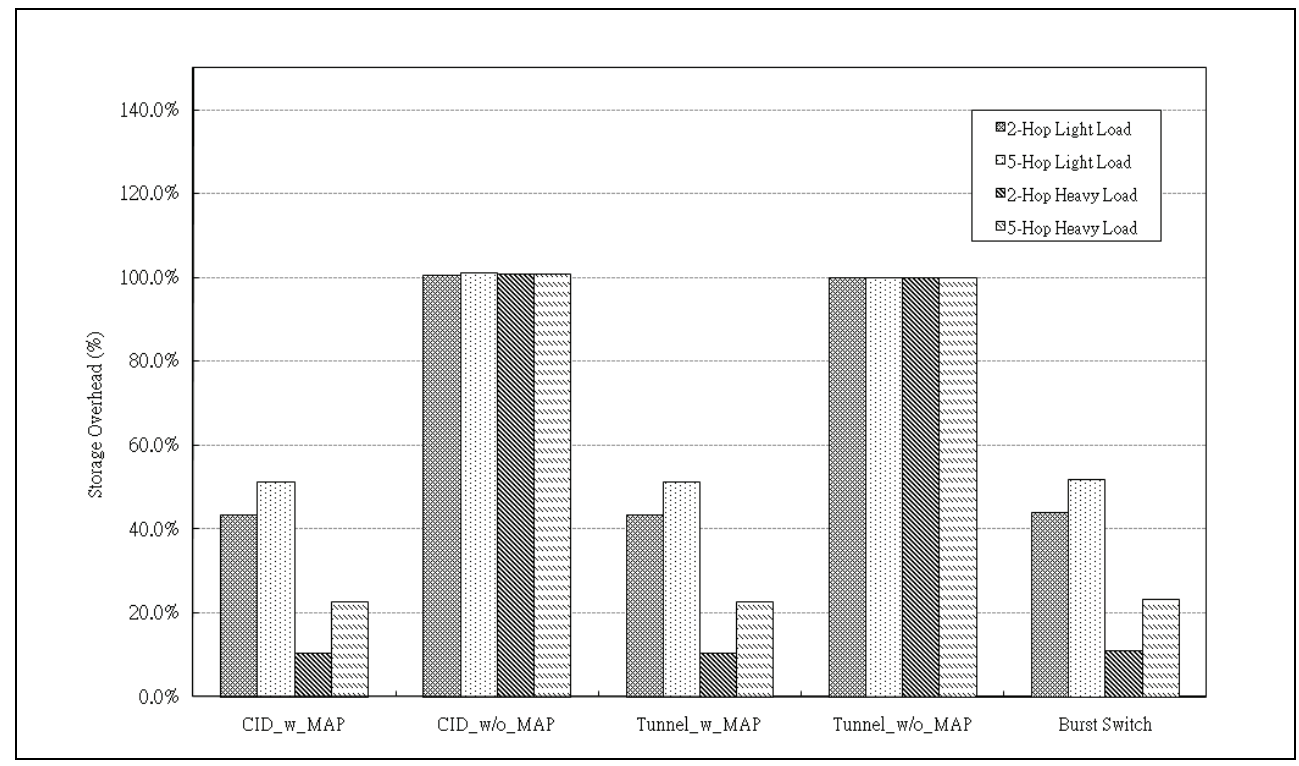

Fig. 15. Overall Comparison for Storage Overhead 


\begin{tabular}{|l|c|c|c|c|}
\hline \multirow{2}{*}{ Schemes Scenario } & \multicolumn{2}{c|}{ 2-Hop } & \multicolumn{2}{c|}{ Multi-Hop } \\
\cline { 2 - 5 } & Processing & Storage & Processing & Storage \\
\hline CID_w_MAP & Good & Good & Bad & Good \\
\hline CID_w/o_MAP & Bad & Bad & Bad & Bad \\
\hline Tunnel_w_MAP & Good & Good & Bad & Good \\
\hline Tunnel_w/o_MAP & Relevant & Bad & Relevant & Bad \\
\hline Burst Switch & Good & Good & Good & Good \\
\hline
\end{tabular}

Table 2. Evaluation Summary

After comparing all the results from the view point of efficiency in processing and storage, the suggestion for employing data forwarding mechanism can be obtained in TABLE II. In CID-based mechanisms, it is suggested that adopting MAP helps on reducing process and storage overheads. However, both schemes do nothing with the processing problem in multi-hop circumstances. For tunnel-based solutions, network operator shall consider deployment scenario to enable MAP or not since tunnel-based scheme with MAP support performs irrelevantly while disabling MAP for tunnel-based solution causes storage problem in multi-hop cases. After all, CID_w_MAP, Tunnel_w_MAP, and Burst Switch are preferred to be used in 2-hop relay networks since the advantage of low processing and buffering overhead were seen. In multi-hop scenario, all the other forwarding schemes are not suggested because these schemes do not perform as superior as Burst Switch. The proposed forwarding can solve all the overhead problems in 2-hop and multi-hop scenarios.

\section{Conclusions}

In this paper, issues for data forwarding in wireless relay networks are discussed and highlighted. It is found that multi-hop relay has the problem of inefficient processing and buffering by analyzing conventional data forwarding schemes in 802.16j MR system. Through the discussions, introducing tunnel headers can prevent RSs to process data redundantly while reusing MAPs can avoid buffering data unnecessarily. However, none of the convention schemes solve both the problems at the same time.

A new simple forwarding scheme is brought out to tackle the addressed issues. The concept of burst switch is provided with end-to-end relay indications, and RSs can filter data efficiently by the proposed burst CID. Furthermore, data associated with the CID are switched between RSs with simple CID matching. The performance evaluations also prove stable and superior performances of the proposed scheme in various network and traffic environments. Comparing with prior mechanisms, burst switch scheme also shows benefits in both 2-hop and 5-hop cases. As a result, it can conclude that relaying with the proposed method provides simple and efficient data forwarding in wireless multi-hop relay networks.

\section{Future research}

Apart from the overhead issues, forwarding data in a wireless relay network will suffer from high packet error rate. The issue is also crucial for a wireless multi-hop network since packets during relay will be corrupted due to unreliable wireless medium. It is expected that data forward in multi-hop environments would make the situation worse. Although 
Automatic Repeat reQuest (ARQ) mechanism is proposed to maintain the wireless communications reliability, it has been shown that conventional ARQ (Fairhurst \& Wood, 2002) cannot overcome the packet error problem efficiently in wireless multi-hop circumstances. Moreover, how a RS participates in error control would impact data forwarding performance directly. Based on error control behaviours, the relay ARQ mechanisms can be classified into three types and are shown in figure 16. The first type is End-to-End Relay $\mathrm{ARQ}$, in which RSs relay packets toward a destination and involve nothing on controlling errors between BS and SSs. The second one is Hop-by-Hop Relay $\mathrm{ARQ}$, and RSs in the scheme trigger feedbacks and retransmissions in per-hop basis. The last type is Two-Link Relay ARQ, which divides an end-to-end transmission into two links and maintains the communications in the links separately. To utilize limited radio resource and provide enhanced relay services, relay ARQ transmissions shall be investigated in the future.

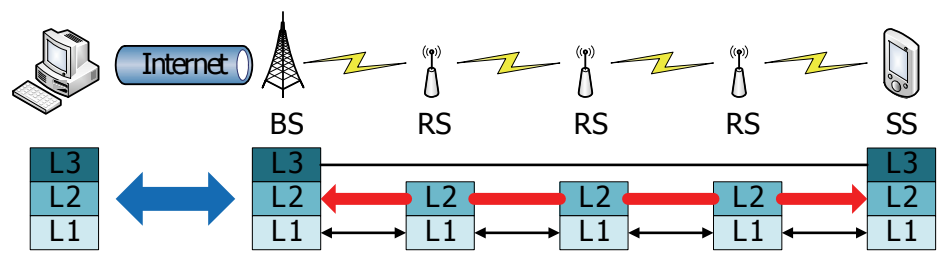

(a) End-to-End ARQ

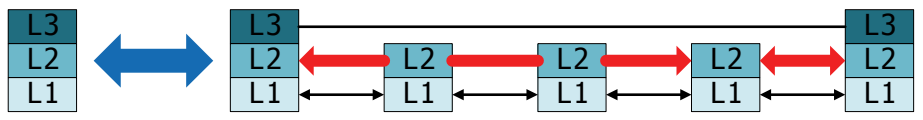

(b) Two-Link ARQ

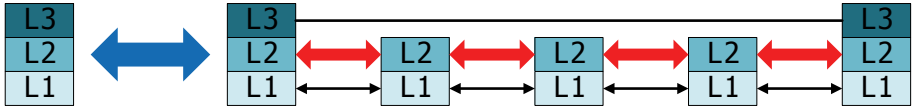

(c) Hop-by-Hop ARQ

Fig. 16. Wireless Relay ARQ Transmissions

\section{References}

Nosratinia, A. ; Hunter, T.E. ; Hedayat, A. (2004). Cooperative communication in wireless networks, IEEE communication magazine, Vol. 42, Issue 10, Oct. 2004, pp. 74-80.

Mohr, W. (2005). The WINNER (wireless world initiative new radio) project-development of a radio interface for system beyond 3G, Proceedings of Personal, Indoor and Mobile Radio Communications, pp. 501-504, July 2005, Berlin, Germany.

Doppler, K. ; Osseiran, A. ; Wodczak, M. ; Rost, P. (2007). On the Integration of Cooperative Relaying into the WINNER System Concept, Proceedings of Mobile and Wireless Communications Summit 2007, pp. 1-5, July, 2007, Budapest, Hungary.

Soldani, D. ; Dixit, S.(2008). Wireless relays for broadband access [radio communications series], IEEE communication magazine, Vol. 46, Issue 3, March 2008, pp. 58-66. 
Tao, Z. ; Teo, K.H. ; Zhang, J.(2007). Aggregation and concatenation in IEEE 802. 16j mobile multihop relay (MMR) networks, Proceedings of IEEE Mobile WiMAX Symposium, pp. 85-90, March 2007, Orlando, Florida.

IEEE Std 802.16e $\mathrm{e}^{\mathrm{TM}}-2005$. IEEE Standard for Local and Metropolitan Area Networks - Part 16: Air Interface for Fixed Broadband Wireless Access System, Amendment 2: Physical and Medium Access Control Layers for Combined Fixed and Mobile Operation in Licensed Bands, IEEE Computer Society and the IEEE Microwave Theory and Techniques Society, February 2006.

IEEE Std 802.16jTM-2009. IEEE Standard for Local and Metropolitan Area Networks - Part 16: Air Interface for Fixed Broadband Wireless Access System, Amendment 1 :Multiple Relay Specification, IEEE Computer Society and the IEEE Microwave Theory and Techniques Society, June 2009.

Fairhurst, G.; Wood, L. (2002). Advice to Link Designers on Link Automatic Repeat reQuest (ARQ), RFC3366, IETF Network Working Group, August 2002.

Wang, S.H. ; Yin, H.C. ; Sheu, S.T. ; Chang, C.S. (2009). Efficient Data Foorwarding Schemes for IEEE 802.16j Multi-hop Relay Networks, Proceedings of Mobile WiMAX Symposium 2009, pp. 24-29, July 2007, CA, US.

Chun, Nie.; Korakis, T. ; Panwar, S. (2008). A Multi-Hop Polling Service with bandwidth Request Aggregation in IEEE 802.16j Networks, Proceedings of Vehicular Technology Conference, 2008, pp. 2172-2176, May 2008, Singapore.

Te, Z. ; Hua, Y. ; (2005). On Link Layer Policies of Data Forwarding over Wireless Relays, Proceedings of Military Communications Conference, pp. 2138-2144, Oct. 2005, Atlantic, US.

Wirth, T.; Venkatkumar, V.; Haustein, T.; Schulz, E.; Halfmann, R.; (2009). LTE-Advanced Relaying for Outdoor Range Extension, Proceedings of Vehicular Technology Conference, pp. 1-4, September. 2009, Anchorage, Alaska, USA.

Kyungmi, P.; Kang, C.G.; Chang, D.; Song, S.; Ahn, J.; Ihm, J.; (2009).Relay-enhanced Cellular Performance of OFDMA-TDD System for Mobile Wireless Broadband Services, Proceedings of Computer Communication and Networks, pp. 430-435, August 2009, Hawaii, US.

Zhao, Z. ;Fang, X. ;Long, Y. ;Hu,Z. ; Zhao, Y. ;Liu. Y. ;Chen, Y. ; Qu, H. Xu, L. ;(2010). Cost Based Local Forwarding Transmission Schemes for Two-Hop Cellular Networks, Proceedings of Vehicular Technology Conference, pp. 1-5, May 2010, Teipei, Taiwan. 


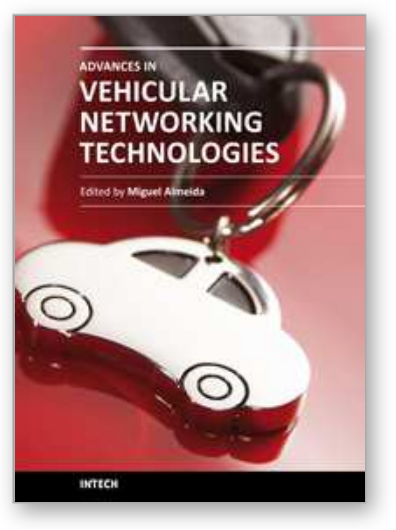

\author{
Advances in Vehicular Networking Technologies \\ Edited by Dr Miguel Almeida
}

ISBN 978-953-307-241-8

Hard cover, 432 pages

Publisher InTech

Published online 11, April, 2011

Published in print edition April, 2011

This book provides an insight on both the challenges and the technological solutions of several approaches, which allow connecting vehicles between each other and with the network. It underlines the trends on networking capabilities and their issues, further focusing on the MAC and Physical layer challenges. Ranging from the advances on radio access technologies to intelligent mechanisms deployed to enhance cooperative communications, cognitive radio and multiple antenna systems have been given particular highlight.

\title{
How to reference
}

In order to correctly reference this scholarly work, feel free to copy and paste the following:

Tzu-Ming Lin, Wen-Tsuen Chen and Shiao-Li Tsao (2011). Data Forwarding in Wireless Relay Networks, Advances in Vehicular Networking Technologies, Dr Miguel Almeida (Ed.), ISBN: 978-953-307-241-8, InTech, Available from: http://www.intechopen.com/books/advances-in-vehicular-networking-technologies/dataforwarding-in-wireless-relay-networks

\section{INTECH}

open science | open minds

\section{InTech Europe}

University Campus STeP Ri

Slavka Krautzeka 83/A

51000 Rijeka, Croatia

Phone: +385 (51) 770447

Fax: +385 (51) 686166

www.intechopen.com

\section{InTech China}

Unit 405, Office Block, Hotel Equatorial Shanghai

No.65, Yan An Road (West), Shanghai, 200040, China

中国上海市延安西路65号上海国际贵都大饭店办公楼 405 单元

Phone: +86-21-62489820

Fax: +86-21-62489821 
(C) 2011 The Author(s). Licensee IntechOpen. This chapter is distributed under the terms of the Creative Commons Attribution-NonCommercialShareAlike-3.0 License, which permits use, distribution and reproduction for non-commercial purposes, provided the original is properly cited and derivative works building on this content are distributed under the same license. 